\title{
Research of the problems and development of the indicators of controllability analysis of pipeline systems
}

\author{
Nikolay Novitsky ${ }^{1, *}$ \\ ${ }^{1}$ Melentiev Energy Systems Institute of Siberian Branch of the Russian Academy of Sciences (ESI SB \\ RAS), Pipeline Systems Department, 130, Lermontov str., Irkutsk, Russia, 664033
}

\begin{abstract}
The article is devoted to the problem of analyzing the controllability of pipeline systems (PLS) of the power industry (heat, water, gas, etc.). Controllability for the first time is considered as a complex property that includes such private properties as permissibility, reliability, and efficiency of PLS operation conditions achieved by the control. Against the background of the general characteristics of the processes of the intelligent automation of PLS management, the urgency of the problem of quantifying the resulting systemic effects is revealed. A brief structuring of the goals and objectives of the analysis of PLS controllability is given. Probabilistic models of their operation conditions are introduced, taking into account the uncertainty of external influences and the internal state of the PLS. A system of probabilistic indicators for an integral quantitative assessment of the controllability of the PLS, which can be used as a basis for analyzing the potential effects of introducing various components of the PLS intellectualization, as well as developing controllability standards is proposed.
\end{abstract}

\section{Introduction}

At present, in economically developed countries, the concept of intelligent networks (Smart Grid) [1-3 and others] is being actively studied in depth, including pipeline systems (PLS) for various purposes (heat, water, gas, etc.) [4-7 and others]. The main goal of PLS intellectualization is to obtain a fundamentally new platform that ensures the harmonization of the requirements and capabilities of all parties involved in the processes of obtaining, transport and consumption of the target product (water, gas, thermal energy, etc.). The analysis of the PLS intellectualization problems [8] shows the focus on ensuring the following basic conditions: 1) dynamic pricing systems that encourage consumers to change their usual consumption schedules; 2) a common information space, as the main system-forming factor responsible for the observability of the processes of production, distribution and consumption for all participants in these processes; 3) a high level of controllability of the system, as the main way to harmonize the requirements of consumers and producers.

\footnotetext{
*Corresponding author: pipenet@isem.irk.ru
} 
The theory of intellectualization of PLS is still in its infancy and does not allow quantifying the potential benefits of the transition to this platform. The focus is on the methods of optimal planning and management of PLS [9-14 and others]. However, there are practically no works devoted to the special study of the problems of PLS technological controllability and intellectualization as a degree of increasing of this controllability. This is largely due to the lack of controllability indicators and methods for their evaluation. Especially important is the assessment of synergistic (systemic) effects from the combined use of different components of intellectualization.

The article deals with the issues of quantitative assessment of controllability. Against the background of a brief description of previously obtained results in this area [15], the main attention is paid to considering this property as complex.

\section{Analysis and formulation of the problem}

The issue of controllability is associated with the feasibility of management. The concept of controllability first appeared in the variations calculus, and then was introduced into the modern theory of optimal control along with the concept of observability. A joint consideration of both concepts, their study, formalization and proof of duality as applied to linear dynamical systems was first conducted by Kalman [16,17]. A brief review of existing concepts of controllability in the theory of optimal control, the theory of reliability of energy systems, and management of engineering networks is given in [15].

In the context of the problems of the intellectualization of the PLS, it is suggested that controllability means possibility to achieve the goals of management with the help of control tools [15]. The difference between this definition and the generally accepted one is that controllability means the possibility of attaining the necessary (required, permissible, useful), and not any states.

The analysis of controllability can be differentiated depending on: 1) its level for qualitative and quantitative; 2) conditions on a priori or a posteriori (in hypothetical conditions or according to retrospective data); 3) the required details on the integral or differential.

Special actualities are issues of a priori integrated assessment of controllability, especially as an integral part of the problem of its synthesis. It seems that in this context, the issue of controllability of PLS has not been studied enough, because it has a multidimensional nature. Among the main aspects of its consideration can be identified target, temporal, spatial and informational. They are conditioned, respectively, to multicriteriality of management tasks, the distribution of management processes in time and geographically, the uncertainty of information. Below there are the issues of quantitative assessment of controllability, taking into account informational and target aspects.

\section{Probabilistic models of controlled states of PLS}

In real conditions, the change in the PLS states in time is a random process, the individual implementations of which almost never repeat. Therefore, the assessment of the consequences of control can be expressed as a function of the distribution of the control results without reference to the target aspect.

States changes are influenced by three main factors: 1) regular environmental exposure (vector $\mathbf{G}$ ); 2) targeted control (vector $\mathbf{u}$ ); 3) sudden changes in the internal state of the PLS of a random nature. Analysis of the impact on the operation of the last type PLS (perturbation) of disturbance (equipment failures, accidents, etc.) is the subject of reliability theory and is not considered here. 
Uncertainties are not only related to the randomness of environmental parameters ( $\mathbf{G}$ ), but also with approximation of information about the PLS elements parameters (vector $\boldsymbol{\alpha}$ ), specifying the internal technical condition of the PLS. Parameters that simultaneously belong to both the model and the external environment are called boundary conditions. With that, it is possible to decompose the state parameters $\mathbf{R}=\{\mathbf{Y}, \mathbf{G}\}$. Respectively, the controlled flow distribution equations can be written as $\mathbf{U}(\mathbf{Y}, \mathbf{G}, \mathbf{u}, \boldsymbol{\alpha})=\mathbf{0}$ or $\mathbf{Y}=\boldsymbol{\varphi}(\mathbf{G}, \mathbf{u}, \boldsymbol{\alpha})$, where $\boldsymbol{\varphi}$ is implicit vector-function that uniquely determines the values of dependent state parameters $\mathbf{Y}$ for given values of vectors $\mathbf{G}, \mathbf{u}$ and $\boldsymbol{\alpha}$. The introduced functional dependence reflects a deterministic causal relationship between the parameters of controlled flow distribution.

We will consider vectors $\mathbf{G}, \boldsymbol{\alpha}$ random, and obeying the normal distribution law with parameters obtained as a result of measurement, foreast or identification. That is $\mathbf{G} \sim N_{m g}\left(\hat{\mathbf{G}}, \mathbf{C}_{G}\right)$ и $\boldsymbol{\alpha}-N_{n a}\left(\hat{\boldsymbol{\alpha}}, \mathbf{C}_{\alpha}\right)$, where $\hat{\mathbf{G}}=E(\mathbf{G})$ is mathematical expectation $\mathbf{G}$; $\hat{\boldsymbol{\alpha}} \approx E(\boldsymbol{\alpha})$ is vector of parameters estimation $\boldsymbol{\alpha} ; \mathbf{C}_{G}=E\left(\boldsymbol{\xi}_{G} \boldsymbol{\xi}_{G}^{T}\right), \mathbf{C}_{\alpha}=E\left(\boldsymbol{\xi}_{\alpha} \boldsymbol{\xi}_{\alpha}^{T}\right)$ are covariance matrices; $\xi_{G}=(\mathbf{G}-\hat{\mathbf{G}})$ is random deviation vector $\mathbf{G}$ from its mathematical expectation; $\boldsymbol{\xi}_{\alpha}=(\boldsymbol{\alpha}-\hat{\boldsymbol{\alpha}})$ is vector estimation errors $\boldsymbol{\alpha} ; m g=\operatorname{dim}(\mathbf{G}) ; n a=\operatorname{dim}(\boldsymbol{\alpha})$, $\operatorname{dim}(\cdot)$ is dimension of vector. Without loss of generality the identification results will be considered uncorrelated with stochastic boundary conditions and the control vector will be determined.

Respectively, the probabilistic flow distribution model can be represented as:

$$
\begin{gathered}
\mathbf{U}(\mathbf{Y}, \mathbf{G}, \mathbf{u}, \boldsymbol{\alpha})=\mathbf{0} \Leftrightarrow \mathbf{Y}=\boldsymbol{\varphi}(\mathbf{G}, \mathbf{u}, \boldsymbol{\alpha}), \\
\mathbf{G} \sim N_{m g}\left(\hat{\mathbf{G}}, \mathbf{C}_{G}\right), \quad \boldsymbol{\alpha}-N_{n a}\left(\hat{\boldsymbol{\alpha}}, \mathbf{C}_{\alpha}\right), \quad E\left[(\mathbf{G}-\hat{\mathbf{G}})(\boldsymbol{\alpha}-\hat{\boldsymbol{\alpha}})^{T}\right]=\mathbf{0} .
\end{gathered}
$$

In many practical cases, the nonlinear distortion of the distribution $\boldsymbol{\varphi}(\mathbf{G}, \mathbf{u}, \boldsymbol{\alpha})$ can be neglected, which allows to approximate it with a normal (Gaussian) distribution with acceptable accuracy. Then the problem is reduced to determining the parameters of this distribution: $\hat{\mathbf{Y}}=E(\mathbf{Y})$ and $\mathbf{C}_{Y}=E\left(\xi_{Y} \xi_{Y}^{T}\right)$, where $\xi_{Y}=(\mathbf{Y}-\hat{\mathbf{Y}})$. Considering $\mathbf{u}$ as a given deterministic vector, we realize linearization (1) in the vicinity of the initial $\{\hat{\mathbf{G}}, \hat{\boldsymbol{\alpha}}\}$ : $\mathbf{Y} \approx \boldsymbol{\varphi}(\hat{\mathbf{G}}, \hat{\mathbf{a}})+\frac{\partial \boldsymbol{\varphi}}{\partial \mathbf{G}}(\mathbf{G}-\hat{\mathbf{G}})+\frac{\partial \boldsymbol{\varphi}}{\partial \boldsymbol{\alpha}}(\boldsymbol{\alpha}-\hat{\boldsymbol{\alpha}})$. Applying to this expression the expectation operation, we get

$$
\hat{\mathbf{Y}} \approx \varphi(\hat{\mathbf{G}}, \hat{\boldsymbol{\alpha}})
$$

At that $\xi_{Y} \approx \frac{\partial \boldsymbol{\varphi}}{\partial \mathbf{G}} \xi_{G}+\frac{\partial \boldsymbol{\varphi}}{\partial \boldsymbol{\alpha}} \xi_{\alpha}$. Hence $\left(\right.$ as $\left.E\left(\xi_{G} \xi_{\alpha}^{T}\right)=\mathbf{0}\right)$

$$
\mathbf{C}_{Y}=E\left(\xi_{Y} \xi_{Y}^{T}\right) \approx \frac{\partial \boldsymbol{\varphi}}{\partial \mathbf{G}} \mathbf{C}_{G}\left(\frac{\partial \boldsymbol{\varphi}}{\partial \mathbf{G}}\right)^{T}+\frac{\partial \boldsymbol{\varphi}}{\partial \boldsymbol{\alpha}} \mathbf{C}_{\alpha}\left(\frac{\partial \boldsymbol{\varphi}}{\partial \boldsymbol{\alpha}}\right)^{T},
$$

The matrix $\mathbf{C}_{Y}$ reflects the degree of uncertainty $\mathbf{Y}$. As can be seen from (4), this matrix is a function of two terms, the last of which reflects the contribution of identifiability [18].

Thus, the analysis of the consequences of control $u$ is reduced to solving the traditional problem of flow distribution (3) from the initial data $\{\hat{\mathbf{G}}, \hat{\boldsymbol{\alpha}}\}$ in combination with the 
additional procedure (4) calculating the covariance matrix $\mathbf{C}_{Y}$ from the given matrices $\mathbf{C}_{G}$, $\mathbf{C}_{\alpha}$, and the derivative matrices $\partial \boldsymbol{\varphi} / \partial \mathbf{G}, \partial \boldsymbol{\varphi} / \partial \boldsymbol{\alpha}$, calculated at a point $\{\hat{\mathbf{G}}, \hat{\boldsymbol{\alpha}}\}$. Examples of introduced vectors, functions, and derivatives for models of steady-state hydraulic state are given in $[15,19]$. The questions of obtaining relations for covariance matrices in relation to other types of flow distribution models (with lumped, variable, and distributed parameters) are given in [20].

\section{Indicators of the PLS controllability}

In the role of the main goals of control, let's emphasize the ensuring of permissibility, reliability and efficiency of states. These requirements are listed in order of importance, as in the absence of the permissible area, consideration of other aspects is meaningless, and the requirement of consumer provision is included in the notion of permissibility. The permissible area in most cases can be represented as bilateral inequality-constraints

$$
\underline{\mathbf{Y}} \leq \mathbf{Y} \leq \overline{\mathbf{Y}} .
$$

From the probabilistic models introduced above, it follows that even with a fixed control the existence of many different states is possible. Thus, in the role of quantitative estimation of the control effects can be used the probability of belonging to the permissible area of states

$$
p(\underline{\mathbf{Y}} \leq \mathbf{Y} \leq \overline{\mathbf{Y}})=p[\underline{\mathbf{Y}} \leq \boldsymbol{\varphi}(\mathbf{G}, \boldsymbol{\alpha}) \leq \underline{\mathbf{Y}}]=\int_{\underline{Y}}^{\bar{Y}} f(\mathbf{Y}) d \mathbf{Y},
$$

where $p$ is probability; $\underline{\mathbf{Y}}, \overline{\mathbf{Y}}$ are the boundaries of the permissible area of a random vector $\mathbf{Y}, f(\mathbf{Y})$ is probability density function.

Even if the mathematical expectation $\mathbf{Y}$ is outside the permissible area (5), there is (perhaps a very small) probability of states implementation belonging to this area and vice versa. Changing the values $\mathbf{u}$, it is got other values $\hat{\mathbf{Y}}, \mathbf{C}_{Y}$, and respectively $p$. Therefore, considering the vector $\mathbf{Y}$ as a random vector-function $\mathbf{Y}(\mathbf{u})$ of an independent deterministic argument, it is possible to influence the probability value (6). Thus, in the role of the integral quantitative assessment of the controllability of the PLS, the value of

$$
p_{a}^{*}=\max _{\mathbf{u}} p(\underline{\mathbf{Y}} \leq \mathbf{Y}(\mathbf{u}) \leq \overline{\mathbf{Y}}),
$$

which we call the maximum probability of belonging to the permissible area.

The problem of controllability analysis by this indicator is reduced to the search for an extremum (7) under the conditions (1), (2) taking into account the restrictions on permissible control

$$
\underline{\mathbf{u}} \leq \mathbf{u} \leq \overline{\mathbf{u}} .
$$

Respectively, various estimates of controllability over a period $T$ can be constructed, for example: the average probability $\frac{1}{T} \int_{0}^{T} p_{a}^{*}(t) d t$ over a period; the time during which the condition $p_{a}^{*}>\delta_{a}$ is satisfied, where $\delta_{\mathrm{a}}$ is the preset threshold of permissible probability; average time of continuous fulfillment of this condition, etc. 
Indicator (7) requires to find such a mathematical expectation of the state $\hat{\mathbf{Y}}(\mathbf{u})$ that is as far as possible from the boundaries of the permissible area, implicitly providing an estimate of how wide or narrow this area is.

The next important indicator, reflecting the aspect of state reliability, is the probability of the state going beyond the permissible limits $\left(p_{r}^{*}\right)$. Even beyond a single state parameter overruns can result in the destruction of equipment, disruption of consumers' supply, the occurrence of water hammer phenomena, cascade development of accidents and other negative consequences, the damage from which is difficult to estimate in advance.

The independent value of the indicator $p_{r}^{*}$ in relation to $p_{a}^{*}$ is determined by the following: 1) with the same probability of belonging to the interval, there may be a different probability of violation of one of its boundaries; 2 ) the probability of simultaneous violation of both boundaries of the interval is zero.

The probabilities of violation of the upper and lower bounds of a single state parameter $Y_{i}$, depending on the control $u$, are defined as:

$$
p_{i}^{+}(\mathbf{u})=p_{i}\left(Y_{i}(\mathbf{u}) \leq \underline{Y}_{i}\right)=\int_{-\infty}^{Y_{i}} f(\mathbf{Y}, \mathbf{u}) d Y_{i}, \quad p_{i}^{-}(\mathbf{u})=p_{i}\left(Y_{i}(\mathbf{u}) \geq \bar{Y}_{i}\right)=\int_{\bar{Y}_{i}}^{\infty} f(\mathbf{Y}, \mathbf{u}) d Y_{i} .
$$

Respectively, for the indicator $\bar{p}_{r}^{*}$ it is proposed to take the minimum of the highest of these probabilities for all the state parameters that can be achieved by control. That is (denoting $n y=\operatorname{dim}(\mathbf{Y}))$

$$
p_{r}^{*}=\min _{\mathbf{u}}\left(\max _{i}\left(\max \left(p_{i}^{+}(\mathbf{u}), p_{i}^{-}(\mathbf{u})\right)\right), i=\overline{1, n y}\right) .
$$

This probability is called the minimal probability of violation of the permissible area. The problem of the controllability analysis by this indicator is reduced to the search for an extremum (9) under the conditions (1), (2) and (8). Note also that the indicator (9) is convenient for normalizing the reliability of the state by setting acceptable values $p_{i}^{+}, p_{i}^{-}, i=\overline{1, n y}$.

When considering the issue of control efficiency, preference is given to natural energy indicators, as the economic ones are related to external price. On the other hand, with indicators of energy efficiency, it is possible always to get their monetary value in a specific price situation.

Introduce into consideration the balance equation for the power of the flows of working medium transported by PLS

$$
N_{I}=N_{N W}+N_{C}^{*}+\Delta N_{C},
$$

where $N_{I}$ is power input from outside PLS; $N_{N W}$ is power loss in pipeline networks; $N_{C}^{*}$ is net power used by consumers; $\Delta N_{C}$ is excess power in the nodes of consumption.

Let's introduce the following integral indicator of the efficiency of the PLS $\eta_{E}=\left(N_{N W}+N_{C}^{*}+\Delta N_{C}\right) / N_{I}$, which has the physical meaning of the efficiency factor of the input power flow. This indicator tends to unity with a decrease in power losses in the network and excess power in the consumption nodes.

The indicator $\eta_{E}$ can be easily transformed into the coefficient of useful use of primary energy resources (electricity, fuel, etc.) through the corresponding characteristics of the efficiency factor of power and power generating equipment $\eta_{I E}=\left(N_{N W}+N_{C}^{*}+\Delta N_{C}\right) / N_{I C}$, 
where $N_{I C}=N_{I C}\left(N_{I}\right)$ is the energy consumption of equipment for creating power flow $N_{I}$.

Each component (10) is a function of the state parameters $R$, including boundary conditions $G$. For example, for a steady-state hydraulic state:

$N_{N W}=\sum_{i \in I_{P L}} y_{i} x_{i}, \quad N_{C}^{*}=\sum_{j \in J_{C}} Q_{j}^{*} P_{j}^{*}, \quad \Delta N_{C}=\sum_{j \in J_{C}}\left(Q_{j} P_{j}-Q_{j}^{*} P_{j}^{*}\right), \quad N_{I}=\sum_{j \in J_{S}} P_{j}\left(Q_{j}\right) Q_{j}+\sum_{i \in I_{P S}} H_{i}\left(x_{i}\right) x_{i}$, $N_{I C}=\sum_{j \in J_{S}} \eta_{j}\left(Q_{j}\right) P_{j}\left(Q_{j}\right) Q_{j}+\sum_{i \in I_{P S}} \eta_{i}\left(x_{i}\right) H_{i}\left(x_{i}\right) x_{i}$, where $J_{S}, J_{C}$ are sets of nodes of flow rate and consumption; $I_{P S}, I_{P L}$ are sets of branches, modeling pumping stations (PLS) and pipelines; $P_{j}$ is pressure in the node $j ; Q_{j}$ is the flow rate in the node for $j \in J_{S}$ and the flow rate of the node for $j \in J_{C} ; x_{i}, y_{i}$ are flow and pressure drop on the branch $i ; H_{i}$ is increment of pressure on the branch with PLS; $Q_{j}^{*}, P_{j}^{*}$ are required (useful), nodal flow rate and pressure for $j \in J_{C}$. Here the boundary conditions include the parameters: $P_{j}, j \in J_{S}$ and $Q_{j}, j \in J_{C}$.

With this, as well as the relationship of the state parameters (1), it is seen that the entered indicator $\eta$ is an implicit function of random arguments and control: $\eta=\eta(\mathbf{G}, \mathbf{u}, \boldsymbol{\alpha})$. Respectively, the problem of the controllability analysis by this indicator is reduced to obtaining the probability density function (as $\eta \sim N\left(\hat{\eta}, \sigma_{\eta}^{2}\right)$ ) for it at the point of the maximum value $\hat{\eta}^{*}$ that can be achieved by control. This problem has the form

$$
\hat{\eta}^{*}=\max _{\mathbf{u}} \eta(\mathbf{Y}(\mathbf{u}), \mathbf{G}) \text { under conditions (1), (2), (5), (8). }
$$

By adding conditions to these limitations $p_{i}^{+}(\mathbf{u}) \geq \delta_{i}^{+}, \quad p_{i}^{-}(\mathbf{u}) \geq \delta_{i}^{-}, \quad i=\overline{1, n y}, \quad$ where $\delta_{i}^{+}, \delta_{i}^{-}$are given thresholds for the probability of violation of the permissible interval limits for each state parameter, it allows carrying out a comprehensive analysis of control efficiency taking into account the requirements for state reliability.

To get the expression for $\sigma_{\eta}^{2}$, it is needed to linearize the implicit $\eta=\eta(\mathbf{G}, \mathbf{u}, \boldsymbol{\alpha})$ dependence in the neighborhood of the solution of problem (11): $\xi_{\eta}=\frac{\partial \eta}{\partial \mathbf{G}} \xi_{G}+\frac{\partial \eta}{\partial \mathbf{u}} \xi_{u}+\frac{\partial \eta}{\partial \boldsymbol{\alpha}} \xi_{\alpha}$. Then $\sigma_{\eta}^{2}=E\left(\xi_{\eta}^{2}\right)=\frac{\partial \eta}{\partial \mathbf{G}} \mathbf{C}_{G}\left(\frac{\partial \eta}{\partial \mathbf{G}}\right)^{T}+\frac{\partial \eta}{\partial \boldsymbol{\alpha}} \mathbf{C}_{\alpha}\left(\frac{\partial \eta}{\partial \boldsymbol{\alpha}}\right)^{T}$, as the value $\mathbf{u}$ is assumed to be deterministic as well $E\left(\boldsymbol{\xi}_{G} \xi_{\alpha}^{T}\right)=\mathbf{0}$.

This shows that the uncertainty of the efficiency indicator $\eta$ is similar to the factors of uncertainty of the state parameters (4), with the same comments about the role of identifiability. Having the parameters of a one-dimensional distribution $N\left(\hat{\eta}, \sigma_{\eta}^{2}\right)$, it is sufficient to simply obtain the probabilities of deviation $\eta$ from a certain value (required, planned, desired), belonging to a certain range, and others. Also at the point of solving the problem (11) it is possible to check the probabilities of the deviation for the optimal power of PLS and its active elements from the stated, established, etc.

\section{Conclusion}

Against the background of a brief description of goals and unfolding processes of the intelligent automation of PLSs, the urgency of the problem of quantitative assessment of the obtained systemic effects is revealed. The problem of estimation of the effects of states 
control under conditions of uncertainty of external influences and the internal state of PLS is considered. A constructive probabilistic approach for such an assessment is proposed. The direct dependence of the uncertainty degree of the control results from the degree of identifiability of PLS is shown. The final expression for this dependence is obtained. The problem of integral quantitative assessment of controllability, which is connected with the multi-criteria control of states in the conditions of information uncertainty, is investigated. For such an assessment, three indicators have been proposed that reflect the main aspects of control: permissibility, reliability and efficiency. The proposed indicators can potentially be used to develop of controllability, as the main cybernetic indicator of the intelligence for PLS.

The research was carried out within the project III.17.4.3 of the Fundamental research program of SB RAS (AAAA-A17-117030310437-4)

\section{References}

1. The concept of the Energy Strategy of Russia for the period up to 2030 (GU IES, Moscow, 2007)

2. “GRID 2030" A National Vision For Electricity's Second 100 Years (Office of Electric Transmission and Distribution of the USA Department of Energy, 2003)

3. European Electric Grids Technology Platform: Electricity Networks of the Future (Official Publications of the European Communities, Belgium, 2006)

4. H. Lund, S. Werner, R. Wiltshire, S. Svendsen, J.E. Thorsen, F. Hvelplund, B.V. Mathiesen, Energy, 68, 1 (2014)

5. C. Temizel, CH. Canbaz, Y. Palabiyik, etc. Proceedings of Middle East Oil \& Gas Show \& Conference, 2019 (2019)

6. Yury Redutskiy, Conceptualization of smart solutions in the oil and gas industry. Procedia Computer Science (Elsevier, 2017)

7. S.V. Mohanasundaram, A. Joyce, Sri Naresh, etc., GIoTS (2018)

8. N.N. Novitsky, Communal services of cities. Series Technical Sciences and Architecture, 101, 456 (2011)

9. Di Nardo, A. Cavallo, M. Di Natale, R. Greco, G.F. Santonastaso, Procedures Engineering, 154, 1275 (2016)

10. Hai Wang, Haiying Wang, Zhou Haijian, Tong Zhu, Energy, 138, 1247 (2017)

11. E. Guelpa, C. Toro, Ad. Sciacovelli, R. Melli, E. Sciubba, V. Verda, Energy, 102, 586 (2016)

12. Mattias Vesterlund, Jan Dahl, Energy Conversion and Management, 89, 555 (2015)

13. M.G. Sukharev, R.V. Samoilov, Analysis and control of stationary and non-stationary states of gas transportation (RGU Oil and Gas, Moscow, 2016)

14. R.Z. Ríos-Mercado, C. Borraz-Sánchez, Applied Energy, 147, 536 (2015)

15. N.N. Novitsky, Pipeline energy systems: methodological and applied problems of mathematical modeling, 159 (Nauka, Novosibirsk, 2015)

16. R.E. Kalman, Proceedings of the First IFAC Congress, 2, 521 (1961)

17. R.E. Kalman, Bull.Soc.Math.Mexicana, 102 (1960)

18. Nikolay N. Novitsky and Oksana A. Grebneva, Proceedings of MMMAOSDPHS 2018, E3S Web of Conferences, 39, 1 (2018)

19. N.N. Novitsky, O.V. Vanteyeva, Proc. ASMDA, 591 (2017)

20. N.N. Novitsky, Estimation of hydraulic circuits parameters (Science, Novosibirsk, 1998) 